\title{
Impact of the Inferior Right Hepatic Veins on Right Liver Lobe Regeneration in Living-Donor Liver Transplant: 3-Dimensional Computed Tomography Scan Analyses in Donors and Recipients
}

\author{
Riccardo Pravisani, ${ }^{1,2}$ Akihiko Soyama, ${ }^{1}$ Mitsuhisa Takatsuki, ${ }^{1}$ Masaaki Hidaka, ${ }^{1}$ \\ Tomohiko Adachi, ${ }^{1}$ Shinichiro Ono, ${ }^{1}$ Takanobu Hara, ${ }^{1}$ Takashi Hamada, ${ }^{1}$ Susumu Eguchi ${ }^{1}$
}

\begin{abstract}
Objectives: The presence of an inferior right hepatic vein significantly modifies the outflow pattern of the right liver and may have an impact on graft regeneration.

Materials and Methods: Study patients were donors and recipients with 3-dimensional computed tomography scan simulation. We studied the physiologic impact of the inferior right hepatic veins on right liver regeneration in donors of left lobe grafts (step $1 ; n=60$ patients) and the efficacy of a inferior right hepatic vein reconstruction policy based on a vessel caliber $>5 \mathrm{~mm}$ criteria in recipients of right liver grafts (step 2; $\mathrm{n}=38$ patients).

Results: In step 1, the regeneration rate at 1 month was comparable between future liver remnants with inferior right hepatic vein (regeneration rate of $28.3 \%$ ) and without inferior right hepatic vein (regeneration rate of $30.2 \%$ ). In step 2, at 3 months after living-donor liver transplant, we observed no significant differences in regeneration rates among grafts anatomically without an inferior right hepatic vein (regeneration rate of $60.7 \%$ ), grafts with an inferior right hepatic vein but no reconstruction (regeneration rate of $64.9 \%$ ), and grafts with an inferior right hepatic vein and its reconstruction (regeneration rate of $68.1 \%$ ). Within the latter subgroup, grafts with a dominant inferior right hepatic vein showed a greater regeneration of the anterior sector with inversion of the anterior-toposterior sector regeneration ratio compared with right hepatic vein-dominant grafts (inferior right

$\overline{\text { From the }{ }^{1} \text { Department of Surgery, Nagasaki University Graduate School of Biomedical Sciences, }}$ Nagasaki, Japan; and the ${ }^{2}$ General Surgery and Liver Transplant Unit, Department of Medicine, University of Udine, Udine, Italy

Acknowledgements: The authors have no sources of funding for this study and have no conflicts of interest to declare.

Corresponding author: Susumu Eguchi, Department of Surgery, Nagasaki University Graduate School of Biomedical Sciences, 1-7-1 Sakamoto, Nagasaki 852-8501, Japan

Phone:+81958197316 E-mail: sueguchi@nagasaki-u.ac.jp
\end{abstract}

Experimental and Clinical Transplantation (2019) 6: 768-774 hepatic vein-dominant vs right hepatic vein-dominant showed anterior sector regeneration rates of $85.7 \%$ vs $52.1 \% ; P=.02$; anterior/posterior sector regeneration ratio was 2.3 vs 0.6 , respectively; $P=.04$ ).

Conclusions: The reconstruction policy for inferior right hepatic vein with caliber $>5 \mathrm{~mm}$ is effective but should be implemented with computed tomography scan simulation. Right lobe grafts with a dominant inferior right hepatic vein are at higher risk of impaired regeneration due to expected large volumes of hepatic venous congestion if the middle hepatic veins tributaries or the inferior right hepatic veins are not reconstructed and an increased rate of right hepatic vein complications.

Key words: Liver congestion, Liver regeneration, Middle hepatic vein tributaries

\section{Introduction}

The inferior right hepatic vein (iRHV) is an accessory anatomic variant of the right liver, connecting independently to the inferior vena cava (IVC) and mainly draining the right posterior sector. Its prevalence varies according to radiologic and deceased-donor specimen series, but overall it is detected in around $60 \%$ of cases. ${ }^{1}$

The presence of an iRHV has crucial relevance in right living-donor liver transplant (LDLT) because it significantly modulates the venous drainage pattern of the graft. ${ }^{1,2}$ Moreover, an effective management of liver outflow is a fundamental determinant of LDLT outcome. ${ }^{1-3}$ The risk related to an impaired venous drainage is the hepatic venous congestion that exposes the parenchyma to both mechanical injury due to acutely increased sinusoidal pressure and ischemic injury due to impaired portal inflow. ${ }^{4}$ As a result, metabolic dysfunction and impaired regenerative 
capacity may occur. ${ }^{4-6}$ It is reported that veno-occluded regions have approximately $40 \%$ of the maximum function of corresponding regions ${ }^{7}$ and that volume atrophy can persist even in long-term follow-up. ${ }^{2}$

Investigations on outflow management have thus far mainly focused on the middle hepatic vein (MHV) tributaries to segment 5 (V5) and segment 8 (V8), whereas reports on iRHV reconstruction in the right lobe graft (RLG) are scarce., ${ }^{3,8-10}$ Moreover, an iRHVbased analysis of outcomes of RLG regeneration has never been performed. Therefore, in this study, our aim was to examine the impact of presence, drainage volume, and reconstruction of $\mathrm{RHV}$ on regeneration of the future liver remnant (FLR) in donors after extended left hepatectomy and regeneration of RLG without MHV in recipients after LDLT.

\section{Materials and Methods}

This was a retrospective study of prospectively collected data. The study was composed of 2 investigation steps. In step 1, we analyzed the physiologic impact on regeneration of iRHV in donors after extended left hepatectomy. In step 2, we analyzed the impact on regeneration of our iRHV reconstruction policy in recipients after LDLT with RLG without MHV.

\section{Patients}

From May 2006 to October 2016, 194 LDLT procedures were performed at the Nagasaki University Hospital (Nagasaki, Japan). There were 111 extended left lobe grafts and 64 RLGs without MHV.

\section{Step 1}

From October 2010 to October 2016, 64 adult patients underwent extended left hepatectomy for graft donation. Four patients were excluded due to unavailability of a 1-month follow-up computed tomography (CT) scan. Therefore, the study population included 60 patients who were divided into 2 groups: the no iRHV group ( $\mathrm{n}=23$ patients) had FLR without iRHV and the iRHV group $(n=37$ patients) had FLR with iRHV.

\section{Step 2}

From May 2006 to October 2016, 64 adult patients underwent LDLT with a RLG without MHV. Exclusion criteria were patient or graft survival of $<3$ months after LDLT (4 patients excluded) and unavailability of strict 3-month follow-up contrastenhanced CT scan (22 patients excluded).Therefore, the study population included 38 patients who were divided into 3 study groups (Figure 1): the no IRHV group ( $\mathrm{n}=12$ patients) included recipients of grafts without $i R H V$, the no reconstruction group $(n=13$ patients) included recipients of grafts with iRHV that were not reconstructed, and the reconstruction group ( $\mathrm{n}=13$ patients) included recipients of grafts with reconstructed iRHV.

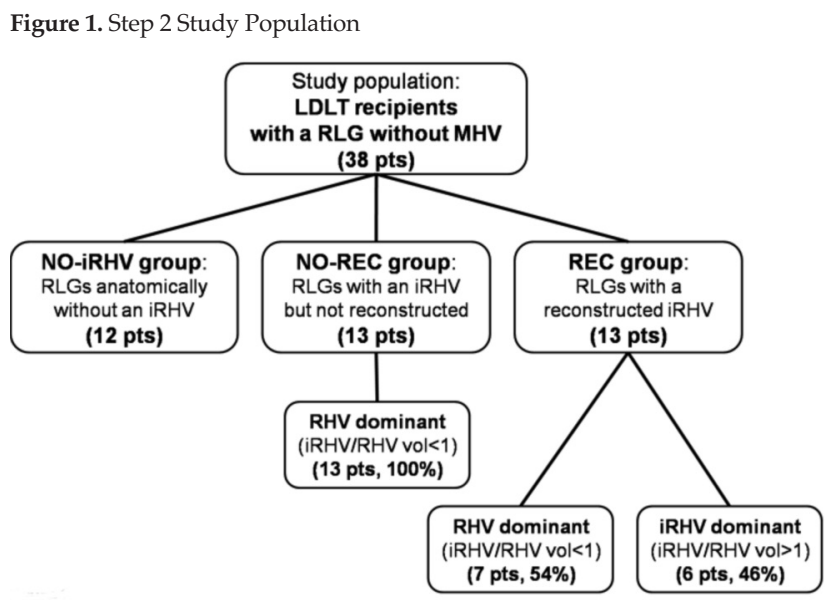

Abbreviations: iRHV, inferior right hepatic vein; LDLT, living-donor liver transplant; pts, patients; REC, reconstruction; RHV, right hepatic vein; RLG, right lobe graft

\section{Preoperative 3-dimensional computed tomography scan analysis}

Image analysis was performed using Synapse VINCENT (Fujifilm Medical, Tokyo, Japan) as previously reported. ${ }^{11,12}$ For reconstruction, $0.5-$ to 3-mm-thick images acquired during a 3-phase (arterial, portal, venous) dynamic study with multidetector CT were used. The stem of the main portal vein was set as a seeding point, and the direction was set for the peripheral side; the portal tree was then extracted automatically. To extract the peripheral thin branches for complete segmentation, a semi-automatic additional setting of seeding points was performed. Each hepatic vein was also extracted using the same procedure from the direction of the confluence to the IVC.

For our study, the iRHV was defined as the drainage vein of the right liver lobe connecting to the IVC independently from the RHV. ${ }^{2}$ Short veins draining just the paracaval area were not classified as iRHV. No distinction was made between iRHV and the right MHV. 
After whole liver volume and vessel reconstruction, we performed virtual liver partition following the course of the MHV as carving transection plane and exposing the vein at the resection surface. The liver segmentation was defined according to the Couinaud classification of liver anatomy.

For donors of extended left livers (step 1), postoperative care included a single routine imaging evaluation at 1 month after graft donation in uncomplicated cases. For RLG recipients (step 2), we evaluated CT scans at 3 months follow-up to minimize the effects of any possible confounding factor on volumetric measurement, such as hepatic venous congestion or early postoperative complications.

\section{Inferior right hepatic vein reconstruction policy and technique}

We performed iRHV reconstruction in recipients when the vessel diameter was larger than $5 \mathrm{~mm}$. The following techniques were alternatively used for reconstruction: direct anastomosis to IVC, patch venoplasty (using recipient's portal vein or umbilical vein), venovenous venoplasty with RHV, or veno-venous venoplasty between multiple iRHVs. These techniques have already been described elsewhere. ${ }^{8,10,13}$

\section{Statistical analyses}

Continuous variables were expressed as means and standard deviation or as median and interquartile range (IQR). For regeneration rate calculation, we compared whole and segmental graft volumes at follow-up examinations after LDLT using the following formula: regeneration rate $=([$ follow-up volume preoperative volume]/ preoperative volume) $\times 100$.

Statistical analyses were performed with SPSS software (IBM SPSS Inc., Chicago, IL, USA). We used $t$ tests, one-way analyses of variance, and MannWhitney tests for analyses of continuous variables and chi-square test or Kruskal-Wallis test for analyses of categorical variables. $P<.05$ was considered significant. We used the Pearson correlation coefficient to determine relationships between continuous variables.

\section{Results}

\section{Step 1}

The study population included 60 donors (42 men and 18 women) with a mean age of $37.7 \pm 12.4$ years. An iRHV was detected in $61.7 \%$ of cases. Multiple iRHVs were identified in $25 \%$ of cases (15 patients).
In these cases, the cumulative volume drainage was calculated. Patient demographic and clinical characteristics are shown in Table 1.

In the iRHV group, the median drainage volume of iRHV was $134 \mathrm{~mL}$ (IQR, 87-213 mL), corresponding to a FLR of $15.6 \%$ (IQR, 10.4\%-21.9\%).The median iRHV-to-RHV drainage volume ratio was 0.25 (IQR, 0.17-0.78), with prevalence of $16.2 \%$ (6/37 patients) of dominant iRHV over the respective RHV (ratio $>1$ ). At 1-month follow-up after donation, the mean liver volume was $1108.02 \pm 208.19 \mathrm{~mL}$, corresponding to a mean regeneration rate of $29.5 \pm 14.0 \%$ (Table 1 ).

We observed no significant differences in regeneration rates between the iRHV group and no iRHV group $(P=.30)$. We also observed no significant differences when the iRHV group was subdivided according to the presence of single or multiple iRHVs $(P=.47)$ or according to a iRHV $/$ RHV $>1$ or $<1(P=.63)$.

\begin{tabular}{|c|c|c|c|c|}
\hline & $\begin{array}{c}\text { Total } \\
(n=60)\end{array}$ & $\begin{array}{l}\text { No iRHV } \\
(n=23)\end{array}$ & $\begin{array}{c}\text { iRHV } \\
(n=37)\end{array}$ & $\begin{array}{c}P \\
\text { Value }\end{array}$ \\
\hline $\begin{array}{l}\text { Age, y } \\
\text { Sex }\end{array}$ & $37.7 \pm 12.4$ & $38.2 \pm 12.9$ & $37.4 \pm 12.3$ & .82 \\
\hline (men:women) & $42: 18$ & $15: 8$ & $27: 10$ & .52 \\
\hline $\mathrm{BMI}, \mathrm{kg} / \mathrm{m}^{2}$ & $22.6 \pm 2.7$ & $23.2 \pm 2.3$ & $22.26 \pm 3.0$ & .20 \\
\hline $\begin{array}{l}\text { SLV, mL } \\
\text { FLR volume, }\end{array}$ & $1226.9 \pm 122.6$ & $1230.2 \pm 120.7$ & $1224.9 \pm 123.5$ & .75 \\
\hline $\begin{array}{l}\mathrm{mL} \\
\mathrm{FLR} \%\end{array}$ & $\begin{array}{l}861 \pm 163.5 \\
65.1 \pm 7.8 \%\end{array}$ & $\begin{array}{r}882.5 \pm 134.8 \\
66.6 \pm 10.1 \%\end{array}$ & $\begin{array}{c}847.6 \pm 179.4 \\
64.2 \pm 5.8 \%\end{array}$ & $\begin{array}{l}.43 \\
.23\end{array}$ \\
\hline $\begin{array}{l}\text { Operative } \\
\text { time, min } \\
\text { 1-month FLR }\end{array}$ & $415.4 \pm 71.1$ & $409.3 \pm 62.3$ & $419.3 \pm 62.3$ & .60 \\
\hline $\begin{array}{l}\text { volume, } \mathrm{mL} \\
\text { 1-month } \\
\text { regeneration }\end{array}$ & $1108.0 \pm 208.2$ & $1125.9 \pm 166.8$ & $1096.9 \pm 231.7$ & .60 \\
\hline rate & $29.5 \pm 14.0 \%$ & $28.3 \pm 14.0 \%$ & $30.2 \pm 14.2 \%$ & 61 \\
\hline
\end{tabular}

Abbreviations: BMI, body mass index; FLR, future liver remnant; iRHV, inferior right hepatic vein; SLV, standard liver volume

\section{Step 2}

Table 2 shows the demographic and clinical characteristics of step 2 recipients and their donors. There were no significant differences between the 3 study groups for the variables analyzed. Of total recipients, 30 were men and 8 were women, with a mean age of $43.0 \pm 13.2$ years. The median Model for End-Stage Liver Disease score was 15.5 (IQR, 12-19), and the prevalence of hepatocellular carcinoma diagnosis was $52.6 \%$. Hepatitis C and hepatitis B virus infections were the cause of underlying liver cirrhoses in 31.6\% and $26.3 \%$ of patients, respectively. Mean operative 


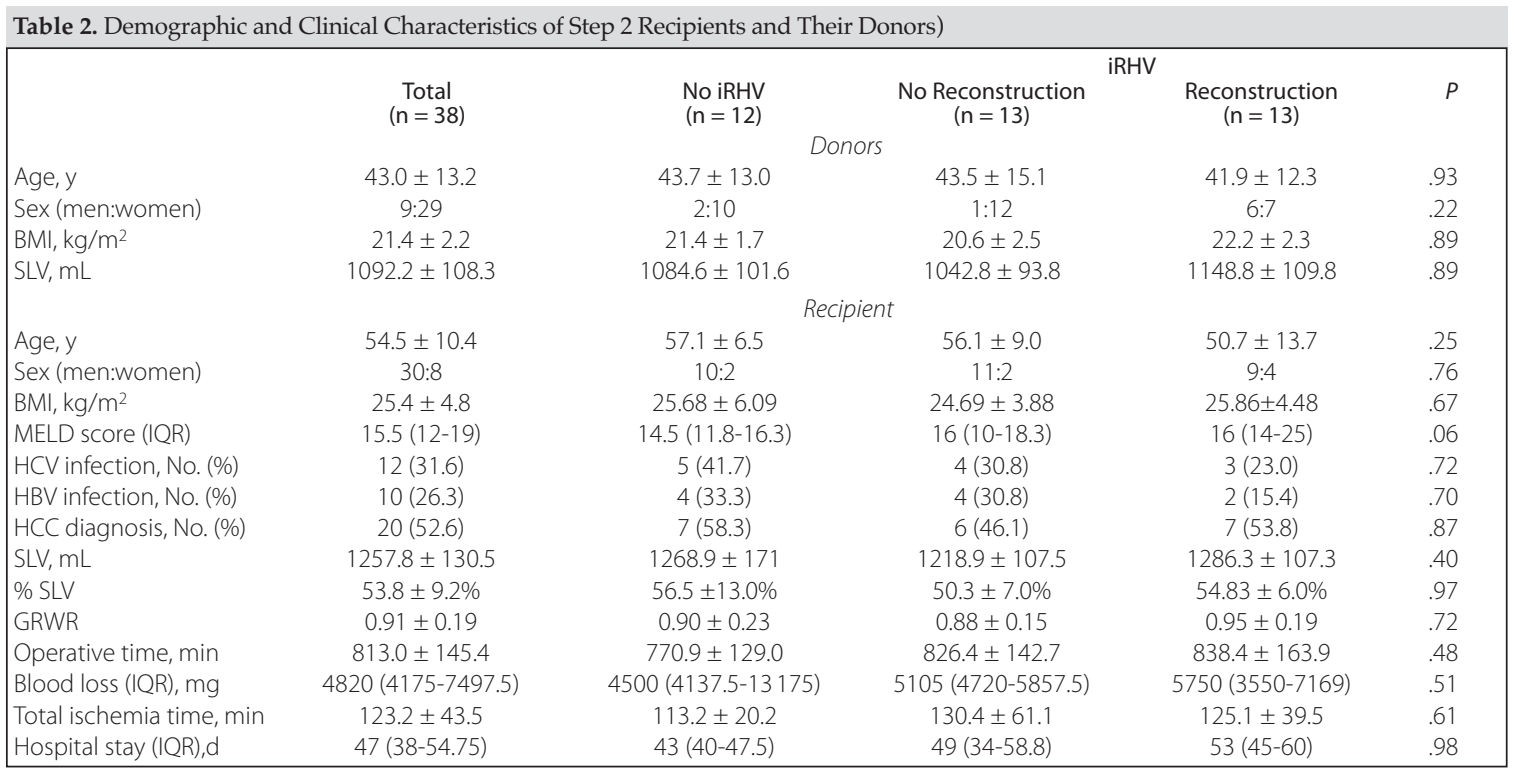

Abbreviations: BMI, body mass index; GRWR, graft-to-recipient weight ratio; HBV, hepatitis B virus; HCC, hepatocellular carcinoma; HCV, hepatitis C virus; IQR, interquartile range; iRHV, inferior right hepatic vein; MELD, Model for End-Stage Liver Disease; SLV, standard liver volume

time was $813.0 \pm 145.4$ minutes, and median length of hospital stay was 47 days (IQR, 38-54.75 d).

\section{Venous drainage patterns}

Single or multiple iRHVs were detected preoperatively in 26 step 2 donors $(68.4 \%)$. When present, the median drainage volume was $12.0 \%$ (range, $6.4 \%-26.3 \%$ ) of the total graft volume, 25.7\% (IQR, 16.8\%-52.0\%) of the posterior sector, and $0.7 \%$ (IQR, $0 \%-3.5 \%$ ) of the anterior sector. Six recipients (15.8\%), all within the reconstruction group, had a dominant iRHV over the respective RHV, as detected at preoperative 3-dimensional CT scan (Figure 2). In such grafts, the median iRHV-to-RHV drainage volume ratio was 1.24 (IQR, 1.1-1.4).

\section{Outflow reconstruction}

Thirteen recipients $(51.8 \%$ of grafts with iRHV) required iRHV reconstruction at liver transplant. The total number of veins detected during preoperative simulation was 19 . Of these, $17(89.5 \%)$ were reconstructed. Techniques applied included direct anastomosis to IVC (6 cases), venoplasty between 2 iRHVs (3 cases), venoplasty with RHV (2 cases), patch venoplasty (1 case), and venoplasty between 2 iRHVs combined with patch venoplasty (1 case). In one graft with 3 iRHVs (total volume drainage $207 \mathrm{~mL}, 28.0 \%$ of the graft volume), only the largest vein $(137 \mathrm{~mL})$ was reconstructed, sacrificing the 2 others $(70 \mathrm{~mL})$. The reconstructed iRHVs had a median drainage volume of $187 \mathrm{~mL}$ (IQR, $125-242.5 \mathrm{~mL})$, corresponding to a median $28 \%$ (IQR 20.3\%-35.2\%) drainage volume of the RLG. This volume was statistically greater compared with volume percentage shown in the 13 cases without iRHV reconstruction $(6.4 \%$; IQR, $4.8 \%-8.6 \% ; P<.01)$.

At the 3-month follow-up, the patency rate of reconstructed iRHVs was $92.3 \%$. One recipient who developed vein occlusion on postoperative day 1 was promptly treated with endovascular stenting. Two recipients in the reconstruction group (overall incidence of 5.3\%) developed a critical stricture of the RHV, which was promptly and effectively treated with endovascular stenting.

Regarding MHV tributary reconstruction, the prevalence in recipients without $\mathrm{R} H \mathrm{~V}$, recipients without reconstruction, and recipients with reconstruction was $25 \%$ (3/12 patients: $1 \mathrm{~V} 5$ and $2 \mathrm{~V} 8$ ), 61.54\% (8/13patients: 1 V5 and 7 V8), and 30.8\% (4/13 patients: $3 \mathrm{~V} 5$ and $1 \mathrm{~V} 8$ ), respectively.

\section{Regeneration}

Three months after liver transplant, the overall regeneration rates for the whole graft volume, the posterior liver sector, and the anterior liver sector were $64.6 \%, 85.8 \%$, and $54.3 \%$, respectively. We observed no significant differences when we compared outcomes among the 3 study groups in 
step 2 (Table 2). However, in the no reconstruction group, we observed an inverse correlation between the percent volume drainage of the non-reconstructed iRHV and regeneration rate of the posterior liver sector (Pearson $\mathrm{r}=-0.64, P=.05$ ).

To further investigate the effects of different venous drainage patterns on RLG regeneration, we divided the reconstruction group into 2 subgroups according to dominancy of either the RHV or iRHV (Figure 2, Table 3). Despite a comparable regeneration rate of the whole graft volume between the 2 subgroups $(P=.97)$, a statistically significant inversion in the segmental regeneration ratio between the anterior and posterior segments $(P=.04)$ was observed. In the iRHV-dominant subgroup, the anterior segment regenerated more than the posterior segment; conversely, in the RHV-dominant subgroup, the posterior segment regenerated more than the anterior segment.

In iRHV-dominant grafts, the percent venous drainage volume of MHV was statistically greater than in RHV-dominant grafts (36.7\% [IQR, 32.6\%-41.8\%] in
iRHV-dominant and $27.2 \%$ [IQR, 26.4\%-30.8\%] in RHV-dominant; $P=.01$ ). Accordingly, the prevalence of MHV tributaries reconstruction in the iRHVdominant subgroup was also higher (3/ 6 patients: $2 \mathrm{~V} 5$ and $1 \mathrm{~V} 8$ ) than in the RHV-dominant subgroup (1/7 patients: 1 V5). On the other hand, the observed cases of RHV stricture (2 patients) and iRHV stricture (1 patient) belonged all to the iRHV-dominant subgroup. Overall, the regeneration rate of the anterior sector was significantly higher in the iRHV-dominant subgroup $(P=.02)$, whereas regeneration rate of the posterior sector was higher in the RHV-dominant subgroup but not at a significant level $(P=.06)$.

\section{Discussion}

Previous reports have already remarked on the importance of iRHV reconstruction in RLG LDLT since the congestion in the related drainage territories may be large enough to be clinically significant. ${ }^{1,3,6,7,10}$ However, the presence of an

Figure 2. Three-Dimensional Computed Tomography Scan Simulation of Different Venous Drainage Patterns in a Right Lobe Graft

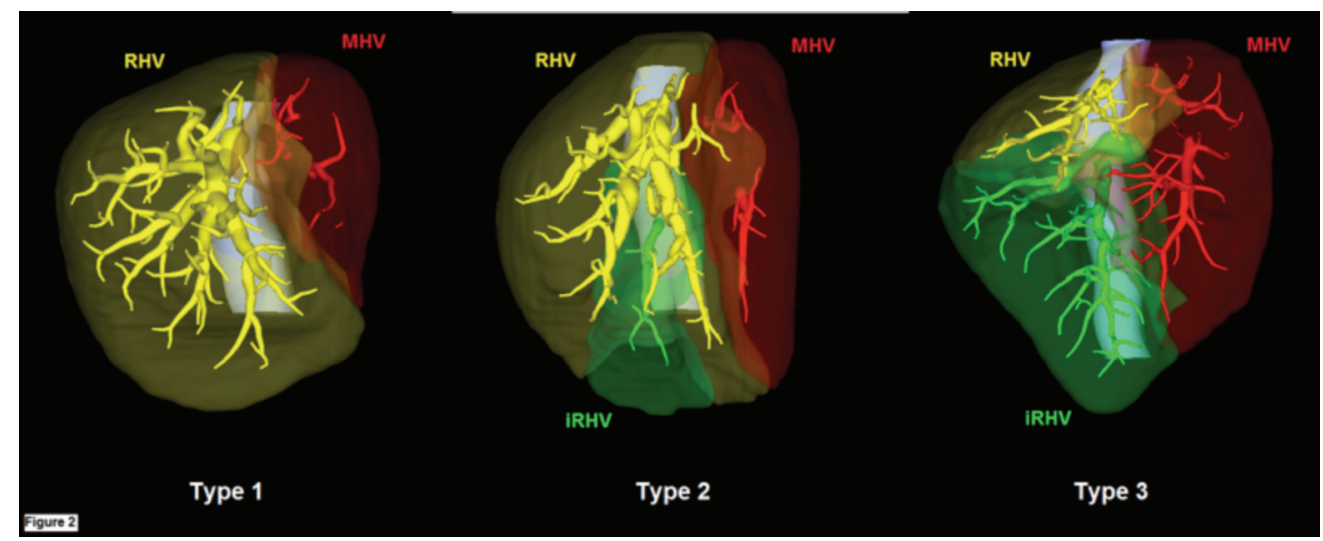

Abbreviations: iRHV, inferior right hepatic vein; MHV, middle hepatic vein; RHV, right hepatic vein

Right lobe graft was classified according to presence and drainage volume of iRHV as follows: type I showed no iRHV, type II showed RHV dominant over respective iRHV, and type III showed iRHV dominant over respective RHV.

Table 3. Regeneration Rates of Right Liver Grafts at 3 Months After Liver Transplant in Step 2 Study Groups and Reconstruction Subgroups

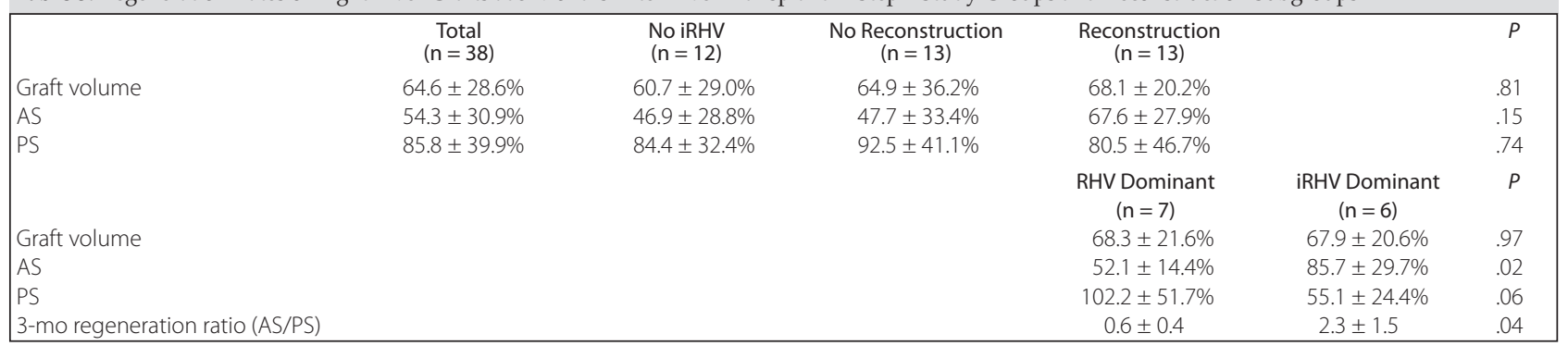

Abbreviations: AS, anterior sector; iRHV, inferior right hepatic vein; PS, posterior sector; RHV, right hepatic vein 
iRHV does actually have wider implications on the overall outflow function at LDLT. It has been speculated that presence of an iRHV ensures multiple outflow pathways for the RLG, which may decrease the risk of critical congestion. ${ }^{3}$ On the other hand, with presence of an iRHV, the associated RHV commonly appears smaller in caliber intraoperatively, thus potentially leading to higher risk of complications at reconstruction. ${ }^{10,14}$ Under this perspective, it becomes relevant and informative to clarify the actual impact of iRHV on the regenerative outcome of the graft.

To rule out whether multiple outflow pathways physiologically guarantee an advantage for the regeneration of the RLG, we first analyzed donors of extended left liver grafts. In this setting, we observed no confounding effects of possible anastomotic complications. On the other hand, the FLR was exposed to congestion due to inclusion of the MHV in the graft; the presence of accessory hepatic veins might have possibly secured a quicker or more effective adaptation to the outflow deprivation. However, no significant differences in regeneration rates were shown between patients with or without an iRHV. This result was used as a reference outcome for our revaluation of regeneration rates in RLG recipient groups with and without iRHV.

There is wide agreement about the use of a diameter larger than $5 \mathrm{~mm}$ as criteria for indication for iRHV reconstruction. $2,8-10,13,14$ In our study, results of step 2 analyses confirmed the validity of this strategy since the regeneration rates among grafts without an iRHV, grafts with an iRHV but not reconstructed, and grafts with a reconstructed iRHV were comparable. Nevertheless, we believe that the use of preoperative 3-dimensional CT volumetry is crucial and should be implemented in the decisionmaking regarding iRHV reconstruction rather than just relying on iRHV caliber, as previously suggested, due to technical reasons. ${ }^{2}$ In our retrospective comparison of percent drainage volume of iRHV in the reconstruction versus the no reconstruction groups, as measured by 3-dimensional CT simulation, we observed significant differences. In addition, in grafts with an iRHV but without reconstruction at LDLT, we observed a negative correlation between the estimated percent drainage volume of the iRHV and the regeneration rate of the posterior sector.

Venous drainage patterns of the right liver can be effectively classified on CT scan volumetry measure- ments according to presence and drainage volume of iRHV, ${ }^{1,2,14}$ in which 3 pattern types are shown. Patients with type I hepatic veins have no iRHV, those with type II hepatic veins have a small iRHV (iRHV $<$ RHV), and those with type III hepatic veins have a large iRHV (iRHV > RHV) (Figure 2). From type I to type III, the percent drainage volumes of iRHV and MHV increase, whereas the percent drainage volume of RHV correspondingly decreases. ${ }^{1-3,14}$ This venous pattern modulation was verified in our study, in which we showed that the percent volume drainage of MHV in those with type III grafts (with a reconstructed dominant iRHV) was statistically greater than in type II grafts (with a reconstructed but not dominant iRHV) and was correctly supported by a higher MHV tributary reconstruction rate.

Reconstructed iRHVs appear to be at low risk of complications even in long-term follow-up and show a superior patency rate versus V5 and V8. Ito and associates reported cumulative patency rates at 1, 3, and 12 months of $98 \%, 93 \%$, and $88 \%$, respectively. Our results at 3 months $(92.3 \%)$ are in line with this report. However, the presence of a large/dominant iRHV (type III venous pattern) predisposes to high risk of RHV complications, which may affect graft regeneration. Our overall RHV complication prevalence of $5.3 \%$ was comparable. ${ }^{3}$ All complications occurred in the iRHV-dominant subgroup, and it may be speculated that the inversion of segmental regeneration ratio between anterior and posterior sectors verified in this subgroups might be a consequence. With this perspective, the anterior sector had significantly greater regeneration and supported adequate regeneration for the whole graft. This may have been due to an effective MHV reconstruction strategy and compensatory hyperactivation induced by a congested hyporegenerating posterior sector. However, the available data did not allow further analyses.

Our present study had several limitations, including its retrospective design, the small size of the study population, and the inability to investigate sectorial regeneration rates of anterior and posterior sectors of FLR in step 1 due to unavailability of contrast-enhanced CT scans of donors with an uneventful postoperative course. Moreover, it was not possible to cross match the impact on regeneration of iRHV and MHV tributary reconstruction due to the small study population size. 


\section{Conclusions}

The presence of an iRHV does not physiologically impact the regeneration of the right liver lobe after partial left hepatectomy. In RLG LDLT, the policy of reconstruction for iRHV with diameter $>5 \mathrm{~mm}$ is effective but should be combined with 3-dimensional CT simulation for venous drainage volume measurements to estimate both the expected congestion volume of MHV and iRHV and to evaluate the dominancy between iRHV and RHV. This strategy would guarantee a tailored reconstructive plan on the specific characteristic of each graft. In particular, the identification of a type III venous pattern should orientate to a more strict and conservative policy not just for iRHV but also for MHV tributaries since the RHV is at higher risk of complications. However, further studies with larger numbers of cases are required to draw definitive management indications.

\section{References}

1. Radtke A, Sotiropoulos GC, Sgourakis G, et al. Hepatic venous drainage: how much can we learn from imaging studies? Anatomic-functional classification derived from three-dimensional computed tomography reconstructions. Transplantation. 2010; 89(12):1518-1525.

2. Uchida K, Taniguchi M, Shimamura T, et al. Three-dimensional computed tomography scan analysis of hepatic vasculatures in the donor liver for living donor liver transplantation. Liver Transpl. 2010;16(9):1062-1068.
3. Ito K, Akamatsu N, Tani K, et al. Reconstruction of hepatic venous tributary in right liver living donor liver transplantation: The importance of the inferior right hepatic vein. Liver Transpl. 2016;22(4):410-419.

4. Kayashima H, Shirabe K, Morita K, et al. Liver regeneration and venous collateral formation in the right lobe living-donor remnant: segmental volumetric analysis and three-dimensional visualization. Transplantation. 2013;95(2):353-360.

5. Chen HL, Tsang LL, Concejero AM, et al. Segmental regeneration in right-lobe liver grafts in adult living donor liver transplant. Clin Transplant. 2012;26(5):694-698.

6. Frericks BB, Kirchhoff TD, Shin $\mathrm{HO}$, et al. Preoperative volume calculation of the hepatic venous draining areas with multidetector row CT in adult living donor liver transplantation: Impact on surgical procedure. Eur Radiol. 2006;16(12):2803-2810.

7. Tani K, Shindoh J, Akamatsu N, et al. Venous drainage map of the liver for complex hepatobiliary surgery and liver transplantation. HPB (Oxford). 2016;18(12):1031-1038.

8. Ito K, Akamatsu N, Togashi J, et al. Outflow reconstruction using cryopreserved homologous venous grafts in living donor liver transplantation. Transplant Proc. 2017:49(1):109-114.

9. Ikegami T, Shirabe K, Yoshiya S, et al. One-step reconstruction of the right inferior hepatic veins using auto-venous grafts in livingdonor liver transplantation. Surg Today. 2013;43(7):769-776.

10. Hwang S, Ha TY, Ahn CS, et al. Reconstruction of inferior right hepatic veins in living donor liver transplantation using right liver grafts. Liver Transpl. 2012;18(2):238-247.

11. Ohshima S. Volume analyzer SYNAPSE VINCENT for liver analysis. J Hepatobiliary Pancreat Sci. 2014;21(4):235-238.

12. Takamoto T, Hashimoto T, Ogata $S$, et al. Planning of anatomical liver segmentectomy and subsegmentectomy with 3-dimensional simulation software. Am J Surg. 2013;206(4):530-538.

13. Lee SG. A complete treatment of adult living donor liver transplantation: a review of surgical technique and current challenges to expand indication of patients. Am J Transplant. 2015;15(1):17-38.

14. Sakamoto Y, Kokudo N, Kawaguchi Y, Akita K. Clinical Anatomy of the Liver: Review of the 19th Meeting of the Japanese Research Society of Clinical Anatomy. Liver Cancer. 2017;6(2):146-160. 